\title{
Sticking the brakes on
}

Neurexins are presynaptic cell adhesion molecules encoded by three genes, each of which encodes a long and short isoform, termed $\alpha$ - and $\beta$-neurexins, respectively. Knockout studies have revealed that $\alpha$-neurexins have a role in neurotransmitter release, but the function of $\beta$-neurexins has been unclear. Südhof and colleagues have now found that $\beta$-neurexins also contribute to neurotransmitter release at excitatory synapses.

The authors found that, in the wild-type adult mouse brain, $\beta$-neurexins are expressed at much lower (10- to 100-fold) levels than $\alpha$-neurexins. Despite the low abundance of $\beta$-neurexins, they set out to examine whether these proteins have a role in synaptic transmission. To do so, they generated conditional knockout (cKO) mice in which the expression of all three $\beta$-neurexins could be 'switched' off.

Compared with wild-type neurons, cortical neurons derived from cKO animals (cKO neurons) exhibited decreased excitatory postsynaptic currents (EPSCs), decreased action potential-dependent presynaptic calcium influx and decreased spontaneous miniature EPSCs (mEPSCs). The impairment in spontaneous mEPSCs was rescued by re-expression of neurexin- $1 \beta$, but not by increased expression of neurexin-1 $\alpha$. This suggests that $\beta$-neurexins are required for normal excitatory neurotransmission, and that modulation of such transmission by $\beta$-neurexins is independent of $\alpha$-neurexin function.

Neuroligin-3, a postsynaptic adhesion molecule that interacts with neurexins, has been shown to have an inhibitory effect on endocannabinoid signalling. This led the authors to hypothesize that $\beta$-neurexins might modulate excitatory transmission in an endocannabinoid-dependent manner. In support of this hypothesis, they found that following treatment with a cannabinoid receptor 1 (CB1) antagonist, control neurons showed a much larger decrease in mEPSC frequency than did cKO neurons. Furthermore, inhibition of 2-arachidonoylglycerol (2-AG; an endogenous cannabinoid produced postsynaptically) synthesis rescued mEPSC frequency in cKO neurons, suggesting that loss of $\beta$-neurexins increased endocannabinoid inhibitory tone via increased 2-AG production.

In slices from cKO mice in which $\beta$-neurexin expression was knocked out in the CA1 hippocampal region, burst-firing pyramidal neurons in the subiculum, but not regularly firing neurons, exhibited a decrease in synaptic strength. Burst-firing cKO neurons also showed a deficit in long-term potentiation. These cell-type-specific synaptic effects were attenuated by application of a CB1 antagonist to the subiculum slices, suggesting that presynaptic $\beta$-neurexins modulate endocannabinoid signalling in vivo.

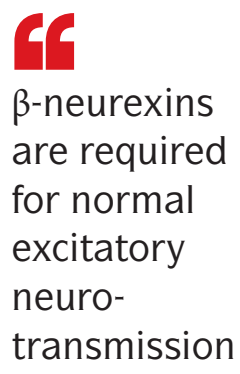

transmission 5
Finally, the authors showed that mice lacking $\beta$-neurexins in CA1 exhibited impaired contextual fear memory. Thus, decreased synaptic strength is accompanied by impaired learning in these animals.

Together, these findings reveal that, despite their relatively low abundance in the brain, $\beta$-neurexins have a unique function in the transynaptic modulation of endocannabinoid tone at excitatory synapses, which is essential for regulating synaptic strength. The endocannabinoid system may thus be a therapeutic target for neuropsychiatric disorders associated with disruptions to synaptic cell adhesion molecules.

\section{Fiona Carr}

ORIGINAL RESEARCH PAPER Anderson, G. R. et al. $\beta$-neurexins control neural circuits by regulating synaptic endocannabinoid signaling. Cell 162, 593-606 (2015)

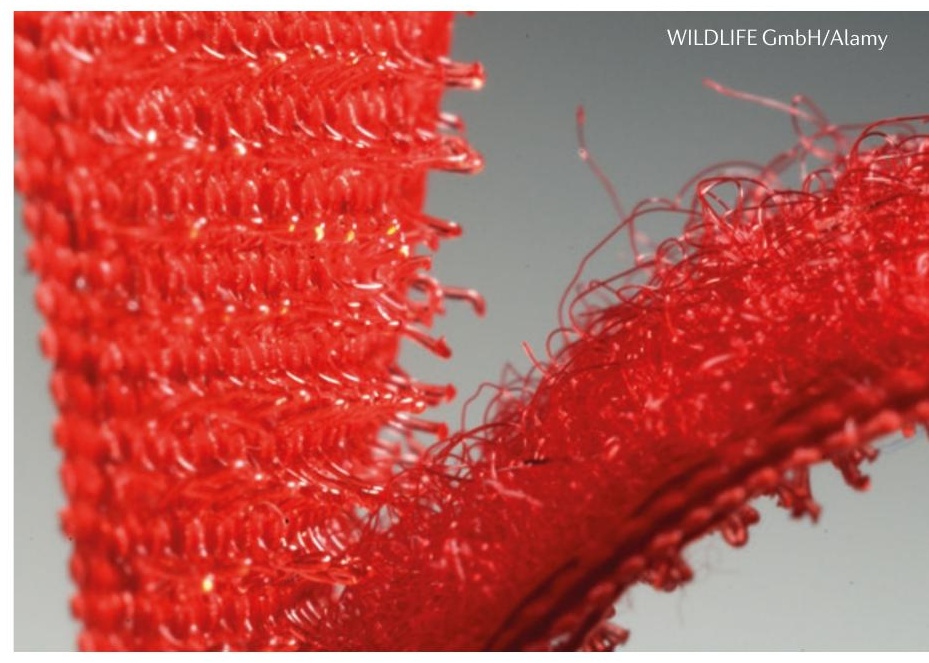

